\title{
Entendendo problemas e conceitos em quadros diferentes da matemática
}

\author{
Paulo Teixeira
}

\begin{abstract}
Resumo
Este trabalho objetiva refletir acerca de questões relativas ao ensino e à aprendizagem de conceitos matemáticos em quadros diferentes, dentro da Matemática. Muitas vezes - particularmente em geometria - quando um problema é proposto há necessidade de se mudar de contexto no qual ele é apresentado de modo a poder resolvê-lo. A essa passagem de um contexto para outro Régine Douady chama de mudança de quadro (Jogo de quadros). Por vezes, fazer uma mudança de quadro pode facilitar a resolução de um problema e/ou ajudar na compreensão de certo procedimento, inclusive quanto à prova de sua validade. Neste trabalho vamos apresentar treze exemplos, em distintos contextos, e relacioná-los às caracterizações acerca do Jogo de quadros, não como necessidade para resolver um problema, mas para mostrar que em outro contexto cada um deles ganha outros olhares, seja para a melhor compreensão dos procedimentos envolvidos, seja para dimensionar o alcance dos conceitos.
\end{abstract}

Palavras-chave: Jogo de quadros; Mudança de quadro; Ensino de Matemática; Conhecimento; Saber.

\section{Abstract}

This work aims to reflect on issues related to the teaching and learning of mathematical concepts in different frameworks within Mathematics. Many times - particularly in geometry - when a problem is proposed there is a need to change the context in which it is presented so that it can be solved. To this passage from one context to another Régine Douady calls a change of framework (Game of frameworks). Sometimes making a change in the framework can facilitate the resolution of a problem and/or help in understanding a certain procedure, including proof of its validity. In this work we present thirteen examples, in different contexts, and relate them to the characterizations about the Game of Frameworks, not as a necessity to solve a problem, but to show that in another context each of them gains other looks, to the procedures involved or to rescale the scope of the concepts.

Keywords: Game of frameworks; Change of framework; Mathematics Teaching; Knowledge; Wisdom. 


\section{Introdução}

O conceito de "quadro, mudança de quadro e jogo de quadros" foi utilizado na didática da matemática francesa, pela primeira vez, por Régine Douady ([3]) na sua tese de doutoramento "Jogo de quadros e dialética ferramenta/objeto".

Segundo [3], um quadro é constituído de "ferramentas" de uma área da Matemática, de relações entre os "objetos", de formulações eventualmente diferentes e de imagens mentais que estão associadas às "ferramentas" em questão. Assim, 2 (dois) quadros podem ter os mesmos "objetos", mas ser diferentes por conta das imagens mentais e da problemática que é proposta para cada um deles [6, p389].

A noção de quadros (mudança de quadros) é importante na resolução de problemas, uma vez que é possível que em diferentes quadros se possam mobilizar diferentes "ferramentas" para resolvê-los. Douady ([3]) propôs essa noção e a "dialética ferramenta objeto" quando observou o trabalho de pesquisadores de matemática em atividade enquanto resolviam problemas.

Segundo Douady ([3]), "[...] na atividade de pesquisa, para resolver um problema, a mudança de quadro, ou seja, uma mudança de contexto ou de modelo teórico é, às vezes, uma mudança decisiva" $[3, \mathrm{p} 54]$.

Para um dado conceito matemático, no contexto da mudança de quadros, distingue-se o conceito "ferramenta" - onde interessa o uso que é feito do conceito para a resolução de um problema (uma "ferramenta" adapta-se aos diversos problemas em que o conceito está presente) do conceito "objeto" - aqui, o conceito está presente dentro de um contexto mais geral, reconhecido que é pela comunidade de educadores e matemáticos [3, p9].

Muitas vezes - particularmente em problemas de geometria - quando um problema é proposto, para resolvêlo é necessário mudar o contexto em que ele é apresentado de modo a poder resolvê-lo. Essa passagem, de um contexto para outro, foi chamado por Douady ([3]) de mudança de quadro (Jogo de quadros).

Por vezes, fazer uma mudança de quadro pode facilitar a resolução de um problema. Mas, também, pode ajudar na compreensão de certo procedimento que por vezes o professor apresenta e faz uso com seus alunos e, por estar em um contexto diferente daquele em que está sendo utilizado, ou então, por necessitar de outros conceitos matemáticos que justifiquem sua validade, o professor se vê impossibilitado de naquele particular contexto poder justificar para seus alunos sua adequação e validade.

Assim, neste caso, embora o procedimento seja adequado para aquele contexto nele o professor não dispõe de razões apropriadas/adequadas - dentro do contexto em que ele está sendo utilizado - para apresentar a prova de sua validade, necessitando, portanto, mudar de quadro (de contexto) para que possa, assim, fazê-lo.

Neste trabalho vamos apresentar exemplos, em diferentes contextos - da Educação Básica e da Álgebra Linear - e relacioná-los às caracterizações presentes no Jogo de quadros.

Mas, estes exemplos serão desenvolvidos não como necessidade para resolver um problema em si, mas para mostrar que em outro contexto cada um deles ganha outros olhares, seja para a melhor compreensão dos procedimentos que foram utilizados no desenvolvimento, seja para dimensionar o alcance dos conceitos apresentados de início. 
É o caso do exemplo de Álgebra Linear, que será apresentado. Nele, conceitos associados às transformações lineares são consolidados mediante uma identificação com conceitos já consolidados da Teoria das Matrizes. Igualmente, conceitos identificados com as transformações lineares também podem ser definidos, de maneira similar, na Teoria das Matrizes. Nestas situações, diz-se que fica estabelecido um isomorfismo (um operador linear bijetor) entre cada matriz de elementos reais e a correspondente transformação linear associada a ela.

A Mudança de quadros é (deveria ser) muito importante no cenário do ensino de Matemática, uma vez que ela contribui para melhorar a compreensão de novos conceitos e procedimentos utilizados ao longo do desenvolvimento de determinado conteúdo matemático.

\section{O que é um "quadro"?}

Douady ([5]) caracteriza a noção de quadro da seguinte maneira: "Um quadro é constituído de objetos de um campo da matemática, de relações entre esses objetos, de suas formulações eventualmente diferentes e das imagens mentais associadas a esses objetos e a essas relações" [5, p135].

Temos como exemplos o quadro algébrico, o quadro geométrico, o quadro numérico, o quadro das funções, o quadro da estatística, o quadro vetorial, o quadro do cálculo numérico, etc. Um quadro pode ser subdividido em outros quadros se o modelo teórico que o define (conjunto de axiomas da teoria) for diferente. Assim, por exemplo, o quadro geométrico pode ser subdividido em quadro da geometria euclidiana, quadro da geometria hiperbólica, quadro da geometria esférica, quadro da geometria projetiva, quadro da geometria afim, quadro da geometria das transformações, quadro da perspectiva cônica, quadro da perspectiva cavaleira, quadro da geometria descritiva, quadro da geometria analítica, etc...

\section{O que é um "mudança de quadro"?}

Segundo Douady ([5]), uma mudança de quadro é uma passagem de um quadro para outro com o propósito de obter formulações diferentes para um problema.

As mudanças de quadro podem ser espontâneas (iniciativa do aluno) ou provocadas (intervenção de outro aluno ou do professor). Essa mudança pode acarretar encontrar novas dificuldades e o funcionamento de "ferramentas e técnicas" não pertinentes na primeira formulação. Utilizamos uma mudança de quadro sempre que num quadro o problema a ser solucionado ou a proposição a ser demonstrada apresenta dificuldades.

Assim, pode-se mudar do quadro da geometria euclidiana para o quadro da geometria analítica ou vice-versa. Outras vezes pode se mudar do quadro das funções para o quadro geométrico. Dependendo do problema proposto, uma mudança de quadro pode ser necessária para obter a solução de uma questão ou pode apenas facilitar a resolução de um problema.

Um jogo de quadros consiste em trabalhar uma mesma questão de Matemática em 2 (dois) diferentes domínios da Matemática. Assim, uma mudança de quadro é a passagem de um quadro para outro e a volta, com o problema resolvido, para o quadro inicial. Os matemáticos, em geral, utilizam o jogo de quadros para contribuir com o entendimento e o caminhar de suas descobertas de resultados e as correlações entre os conceitos que estão presentes em um quadro e em outro.

O avanço nas pesquisas empreendidas por [3] foi o de transportar alguns elementos deste procedimento de mudança de quadro - próprio dos matemáticos - para os alunos da Educação Básica ou 
Superior, com o propósito de contribuir para a melhoria da aprendizagem de novos conceitos e as relações entre diferentes conceitos da Matemática.

Uma mudança de quadro se faz necessária quando existe uma grande dificuldade para resolver um problema ou para demonstrar um resultado em um determinado quadro no qual o conceito ou o problema está sendo apresentado.

Portanto, e de modo a resolver esse impasse, se procura outro quadro onde as "ferramentas" e os "objetos" matemáticos disponíveis facilitam a resolução do problema ou a demonstração e se tenha argumentos que validem os procedimentos utilizados. Depois, uma vez resolvido o problema ou feita a demonstração, se transporta a solução para o quadro inicial.

Porém, de maneira a justificar a necessidade de fazer-se uma mudança de quadro no ensino da Matemática é preciso contar com os conceitos presentes na Teoria dos Registros de Representação Semiótica, de autoria de Raymond Duval ([7]), uma vez que essa teoria é forte aliada neste processo por conta de estar diretamente relacionada com os procedimentos que precisam ser feitos por conta da utilização de novos registros e representações presentes no novo quadro e a correta correlação com os registros e representações do quadro anterior.

Douady ([5]) sugere um jogo interativo entre quadros para fazer progredir os alunos na fase de busca de uma solução para um problema. Esse "Jogo de Quadros" consiste em mudanças de quadro provocadas por iniciativa do professor em certas situações as quais permitem fazer avançar o aluno na resolução do problema. Trata-se do desenvolvimento de um procedimento no qual se transfere o problema de um quadro para outro, interpretam-se as correspondências entre os elementos dos 2 (dois) quadros, resolve-se o problema, e finalmente volta-se com a solução do problema para o quadro de partida.

Nesses procedimentos para a busca da solução do problema, Douady ([3]) distingue 3 (três) fases: o problema deve mobilizar conhecimentos anteriores dos alunos, mas estes conhecimentos são insuficientes para resolver o problema proposto. Assim, será preciso fazer uma transferência de um quadro para o outro, onde nesse último é dada uma interpretação ao fenômeno a ser estudado; um novo conceito deve ser estabelecido no novo quadro para resolver o problema, por meio de correspondências imperfeitas (seja por razões matemáticas ou por conhecimentos insuficientes por parte dos alunos) entre objetos e relações dos dois quadros. Uma nova e adequada "ferramenta" é estabelecida, ampliando os conhecimentos anteriores dos alunos, contribuindo para ampliar a visão conceitual e, finalmente, uma melhoria que se obtém entre essas correspondências - permitindo o progresso do conhecimento em cada um dos quadros quando o professor institucionaliza o novo "objeto" que surgiu das ampliações conceituais, fornecendo ao "objeto" a categoria de um novo "objeto matemático".

Com a conclusão das três fases é possível dimensionar o alcance que a mudança de quadro proporciona para a ampliação dos conceitos conhecidos de início, e a correlação entre eles.

Ademais, a própria compreensão acerca dos novos conceitos e procedimentos que surgem ao longo das fases, e a posterior sistematização e consolidação destes pelo professor, justificam plenamente fazer uma mudança de quadro, quando necessária.

Segundo Duval ([7]), autor da "Teoria dos Registros de Representação Semiótica", os "objetos" de estudo em Matemática recorrem a notações simbólicas, gráficos, tabelas, figuras e esquemas como seus representantes.

Segundo o autor, de modo que se adquira compreensão em Matemática é preciso fazer distinção 
entre o "objeto" e a sua "representação". Com o passar do tempo, havendo dúvidas entre o papel de um e do outro, tal prática acarreta perda da compreensão acerca do conceito matemático envolvido, pois os conhecimentos apropriados tornam-se elementos que destoam dos contextos de aprendizagem - geram ausência de atenção ou passam a ser representações que não têm razão de ser produzidas.

Segundo o autor, "as representações semióticas dos objetos matemáticos são extrínsecas à aprendizagem conceitual dos objetos" [7, p14]. Assim, "para começar em Matemática, as representações semióticas não são somente indispensáveis para fins de comunicação, elas são necessárias ao desenvolvimento da atividade matemática" [7, p15].

Segundo Duval ([7]), uma mudança de quadro é uma mudança de contexto, uma mudança de modelo teórico, uma mudança de direção do pensamento. Daí decorre a importância que se empresta à Teoria dos Registros de Representação Semiótica para que se façam Mudanças de Quadros pertinentes e apropriadas. Segundo Rogalski ([8]), "para certos tipos de objetos alguns registros serão mais adaptados a determinados quadros, às vezes difíceis de distinguir quadro e registro" [8, p15]

Segundo o autor, a noção de ponto de vista é menos precisa que a de quadro e registro, pois se pode dizer que uma mudança de quadro ou de registro é uma ação que está relacionada à mudança de ponto de vista de um "objeto". Por outro lado, é possível que, mesmo mudando de ponto de vista, se permaneça em um mesmo quadro ou em um mesmo registro.

Para Almouloud ([1]), pontos de vista diferentes para um objeto matemático são maneiras diferentes de olhá-lo, de fazê-lo funcionar e, eventualmente, de defini-lo. Nesse sentido, olhar um objeto em diferentes quadros é ter diferentes pontos de vista, embora se possam ter vários pontos de vista dentro de um mesmo quadro ([1, p81]).

\section{Diferentes exemplos de mudanças de quadros}

Por vezes, ao necessitar de outro conceito matemático para justificar a adequação e a validade de alguma afirmação, o professor se vê impossibilitado de naquele particular contexto de resolução de um problema ou durante o desenvolvimento de um conteúdo, fazer isso com argumentos simples e próprios do contexto.

Assim, será preciso lançar mão de um diferente contexto daquele que está sendo utilizado de modo a elucidar a questão posta. Transitar para este novo contexto é fazer uma mudança de quadro. A mudança facilita a resolução de um problema e ajuda na compreensão de um conceito ou de um procedimento e, por essa, o professor ou o aluno deve fazer uso dessa possibilidade/necessidade.

Portanto, ao considerar que o procedimento esteja adequado para aquele contexto, mas não dispondo de razões apropriadas/adequadas, no universo do quadro que está sendo utilizado para garantir a prova da sua validade, é perfeitamente aceitável e, por vezes, imprescindível, que uma Mudança de Quadro seja feita. Apresentamos, a seguir, exemplos em diferentes contextos no universo da Matemática, e os relacionamos com as características presentes no Jogo de Quadros.

Os exemplos que serão desenvolvidos a seguir não têm como necessidade premente ter de fazer uma Mudança de Quadro para resolver o problema em si, mas para mostrar a importância que a mudança de quadro em si tem, uma vez que novo contexto (quadro), cada um dos exemplos ganha um novo olhar - seja para a melhor compreensão dos procedimentos utilizados durante a resolução, seja para ampliar o campo conceitual em relação à questão apresentada de início, permitindo 
identificar as relações entre diferentes conceitos próprios da Matemática e contribuindo para a apropriação de novos conhecimentos matemáticos.

Assim, conhecimentos de conteúdo e conhecimentos pedagógicos de conteúdo devem caminhar juntos no dia a dia da sala de aula com o propósito de contribuir para uma aprendizagem efetiva. Sobretudo, mostra a necessidade de o professor estar preparado para ensinar, em conformidade com as considerações de Shulman (1986) ([9] apud Shulman (1986)).

É o caso do exemplo de Álgebra Linear, segundo o qual os conceitos associados às Transformações Lineares são consolidados mediante a identificação com os conceitos presentes na Teoria das Matrizes, e vice-versa. Nessas situações, fica estabelecido um isomorfismo (um operador linear bijetor) entre uma matriz de elementos reais e a correspondente Transformação Linear associada a ela. Portanto, conceitos e resultados da Teoria das Matrizes podem ser obtidos e provados mediante o desenvolvimento inicial das Transformações Lineares.

Exemplo 1. O quadro da Aritmética de números naturais é constituído da construção do conjunto, de operações elementares e de resultados envolvendo esses elementos. Já o quadro da Teoria dos Conjuntos é constituído por relações de pertinência, de inclusão e operações entre conjuntos, entre outros. Se a axiomática que envolve os números naturais for considerada, o quadro inclui todos os resultados já matematicamente provados.

Para provar que o procedimento de determinação de todos os divisores de um número - bastante utilizado nos livros didáticos da Educação Básica - sempre funciona, mas se torna incipiente para resolver outras situações que têm proximidade com os conceitos envolvidos, será preciso lançar mão da ideia de mudança de quadros, segundo a perspectiva de Douady ([5]).

Inicialmente, se quer resolver o seguinte: Quantos divisores inteiros e positivos possui o número $12 ?$

Uma parcela considerável de professores dos anos iniciais do Ensino Fundamental utiliza o seguinte processo para determinar quais são os divisores de 12 e como calcular o quantitativo desses divisores:

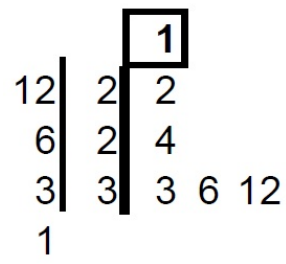

Figura 1: Dispositivo prático para a determinação dos divisores de 12.

É um procedimento canônico bastante conhecido e utilizado pelos professores e, igualmente, está presente em muitos livros didáticos. Da maneira como o procedimento é apresentado e desenvolvido, ele funciona bastante bem para certo tipo de situações que envolvem a determinação de todos os divisores de um número e a totalidade deles por contagem direta, como é o caso do exemplo acima. As questões que se colocam são: Porque esse procedimento funciona? Qual justificativa matemática está presente na sua utilização? 
Depois de dividir o número pelos divisores primos em ordem monótona (iguais ou maiores do que) e, assim, "fatorar" o número dado segundo números primos, por vezes o professor desenha um traço vertical e coloca o número 1 em destaque (Por que razão se faz assim? Porque é assim, costumam os professores referir-se a esse repetido hábito) e, em seguida, multiplica-se os fatores primos do número dado pelos resultados anteriores até que o último primo tenha sido multiplicado pelos anteriores. Por fim, o professor informa a seus alunos que pelo fato de 12 ser escrito como $12=2^{2} \cdot 3^{1}$, então para determinar o quantitativo de divisores de 12 basta tomar os expoentes dos fatores primos do número dado e, um a um, e após somar uma unidade a cada um deles, multiplicar os resultados entre si, obtendo como resultado a totalidade de divisores do número dado. Assim, o total de divisores de 12 é: $(2+1) \cdot(1+1)=3 \cdot 2=6$ divisores. Mas, porque isso sempre funciona? Por que é costume fazer-se assim?

De modo a provar que esta técnica funciona perfeitamente bem vamos fazer uma Mudança de Quadros de maneira a provar que o procedimento é válido e que ele encontra fundamentação teórica matemática que garante sua validação e, portanto, que o resultado é verdadeiro.

De fato: Um número $a$ é divisor de 12 se e somente se $a$ é da forma $a=2^{m} \cdot 3^{n}, m \in\{0,1,2\}$ e $n \in\{0,1\}$, pois $12=2^{2} \cdot 3^{1}$.

Como 1 é divisor de qualquer número diferente de zero, então as escolhas $m=0$ e $n=0$ são as escolhas triviais, ou seja, são as menores escolhas para os expoentes de cada um dos fatores primos do número dado, e, quando combinados pelo produto, resulta no valor 1. Essas escolhas sempre serão possíveis de ser feitas quando se "fatora" o número do qual se deseja conhecer os divisores e para determinar a quantidade de divisores. No exemplo, as escolhas recaem nos fatores primos 2 e 3, a saber: $2^{m} \cdot 3^{n}=2^{0} \cdot 3^{0}=1$. As outras opções de escolhas para os expoentes dos fatores primos 2 e 3 são as seguintes:

$m=1$ e $n=0$, que determina o divisor $2^{m} \cdot 3^{n}=2^{1} \cdot 3^{0}=2 ; m=2$ e $n=0$, que determina o divisor $2^{m} \cdot 3^{n}=2^{2} \cdot 3^{0}=4 ; m=0$ e $n=1$, que determina o divisor $2^{m} \cdot 3^{n}=2^{0} \cdot 3^{1}=3 ; m=1$ e $n=1$, que determina o divisor $2^{m} \cdot 3^{n}=2^{1} \cdot 3^{1}=6 ; m=2$ e $n=1$, que determina o divisor $2^{m} \cdot 3^{n}=2^{2} \cdot 3^{1}=12$. Essas escolhas encerram todas as "combinações" possíveis escolhas entre os fatores primos e os respectivos expoentes que, por sua vez, remete à ideia combinatória, presente em um dos significados do conceito da multiplicação de números naturais.

A tabela de dupla entrada a seguir ilustra as "combinações" que são feitas entre dos fatores primos 2 e 3 e seus possíveis expoentes: 


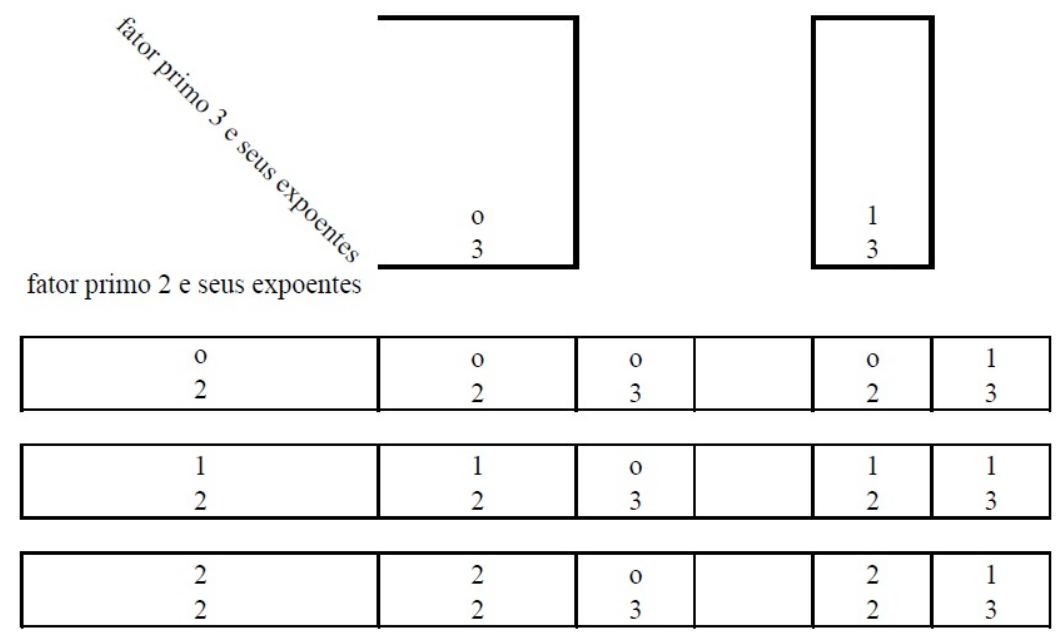

Figura 2: Tabela de dupla entrada com as "combinações" entre os fatores primos de 12 e seus expoentes.

Portanto, o total de divisores do número 12 é 6 (seis). Há 3 modos de escolher o expoente do fator primo 2, consoante sejam as escolhas 0,1 ou 2. Há 2 modos de escolher o expoente do fator primo 3, conforme sejam as escolhas 0 ou 1. Para cada escolha do expoente do fator primo 2 se pode combinar com uma escolha para o expoente do fator primo 3. Portanto, pelo Princípio Multiplicativo (ou Princípio da Multiplicação), da Análise Combinatória, tem-se que o número total de escolhas possíveis é $3 \cdot 2=6$, correspondente ao total de divisores do número 12 .

De modo similar ao procedimento sistemático de colocar o número $1 \mathrm{em}$ destaque (como visto no procedimento anterior), tal fato se justifica pelas escolhas iguais a zero, sempre, dos expoentes de cada um dos fatores primos do número dado, como foi feito na observação anterior quando da escolha de $m=0$ e de $n=0$ (uma escolha imediata, natural). Assim, se um número possui os fatores primos $2,3,5,7,11$, etc, sempre será possível fazer a escolha natural de todos os expoentes iguais a zero para cada um dos fatores primos que estão presentes na fatoração do número dado, o que acarreta encontrar, então, o valor $1=2^{0} \cdot 3^{0} \cdot 5^{0} \cdot 7^{0} \cdot 11^{0} \ldots$

Essa escolha é sempre possível de ser feita uma vez que o conjunto de opções dos expoentes de cada um dos fatores primos de um número é um conjunto de números naturais em que o 0 (zero) é sempre um de seus elementos.

Por outro lado, fica claro que elevar qualquer fator primo do número dado à potência 1 e os demais fatores primos do número à potência zero determina todos os fatores primos do número dado que, obviamente, também são divisores imediatos, do número dado. No exemplo dado tem-se: $2^{1} \cdot 3^{0}=2$ e $2^{0} \cdot 3^{1}=3$ como os divisores primos diferentes de 1 .

Assim, para provar que o procedimento apresentado acima de fato "funciona", será preciso fazer uma Mudança de Quadros para o quadro da Análise Combinatória, onde é possível provar que o "procedimento" em tela nada mais é que um mecanismo para justificar as escolhas do zero como os expoentes dos fatores primos do número dado para determinar o divisor 1, e que os demais divisores do número são obtidos por "combinações" entre as possíveis escolhas entre os expoentes 
dos fatores primos presentes no número.

A prova por meio de conceitos da Análise Combinatória é bastante motivadora, pois ela amplia o campo conceitual no sentido de permitir responder a outras questões dentro do mesmo contexto de divisores de um número que não o seriam apenas com os conhecimentos acerca do procedimento em si como por exemplo, o de decidir se um determinado número é ou não divisor de outro sem que seja preciso determinar todos os divisores do número dado e compará-los com os divisores do número que foi dado, mas a compreensão acerca do modo como o número é escrito como o produto de seus fatores primos elevados aos possíveis expoentes, respondendo à pergunta apenas tomando por base a possibilidade ou não da escolha de todos os expoentes do número que se deseja verificar se é divisor, ou não, para os respectivos fatores primos presentes nos dois números.

Assim, por exemplo, responder se 8 é ou não divisor de 12 se resume a "fatorar" o número 8, o que dá $2^{3}$, e constatar que 3 não é uma possível escolha dentre as opções dos expoentes do fator primo 2 que está presente quando da "fatoração do número 12", que são os expoentes: 0 (zero), 1 (um) ou 2 (dois), pois $12=2^{2} \cdot 3^{1}$. Portanto, 8 não é divisor de 12 , mas 6 é divisor de 12 .

Exemplo 2. Suponha que se está diante da situação de determinar qual o menor número natural que tem 24 divisores. Como proceder? O procedimento apresentado acima, de "fatorar" um número e determinar seus divisores não é suficiente para resolver este problema. Nesse caso, a decisão deve recair sobre as possíveis escolhas dos expoentes dos fatores primos 2, 3, 5 e 7, respectivamente os expoentes $m, n, p$ e $q$, que satisfaçam aos possíveis produtos dos expoentes somados à unidade, conforme: $(m+1) \cdot(n+1) \cdot(p+1) \cdot(q+1)=24$, tomando-se os fatores primos de dois em dois, de três em três ou até os quatro fatores, e decidir sobre quais expoentes determinam o menor número que tem 24 divisores. Assim, possivelmente um aluno começaria a solução desse problema construindo a tabela abaixo (ou outra similar) com a escolha de dois, três ou até quatro dentre os fatores primos $2,3,5$ ou 7 . 


\begin{tabular}{|c|c|c|c|c|l|l|}
\hline $\begin{array}{c}24 \text { escrito } \\
\text { em 2,3 ou } 4 \\
\text { produtos }\end{array}$ & $\begin{array}{c}\text { Expoente } \\
\text { do fator } \\
\text { primo 2 }\end{array}$ & $\begin{array}{c}\text { Expoente } \\
\text { do fator } \\
\text { primo 3 }\end{array}$ & $\begin{array}{c}\text { Expoente } \\
\text { do fator } \\
\text { primo } 5\end{array}$ & $\begin{array}{c}\text { Expoente } \\
\text { do fator } \\
\text { primo } 7\end{array}$ & $\begin{array}{c}\text { Número obtido contendo } \\
24 \text { divisores }\end{array}$ & Situação \\
\hline $24=3.2 .2 .2$ & 2 & 1 & 1 & 1 & $2^{\wedge} 2^{*} 3^{\wedge} 1^{*} 5^{\wedge} 1^{*} 7^{\wedge} 1=420$ & MAIOR \\
\hline $24=2.3 .2 .2$ & 1 & 2 & 1 & 1 & $2^{\wedge} 1^{*} 3^{\wedge} 2^{*} 5^{\wedge} 1^{*} 7^{\wedge} 1=630$ & MAIOR \\
\hline $24=2.3 .4$ & 1 & 2 & 3 & 0 & $2^{\wedge} 1^{*} 3^{\wedge} 2^{*} 5^{\wedge} 3^{*} 7^{\wedge} 0=750$ & MAIOR \\
\hline $24=2.4 .3$ & 1 & 3 & 2 & 0 & $2^{\wedge} 1^{*} 3^{\wedge} 3^{*} 5^{\wedge} 2^{*} 7^{\wedge} 0=1350$ & MAIOR \\
\hline $24=3.2 .4$ & 2 & 1 & 3 & 0 & $2^{\wedge} 2^{*} 3^{\wedge} 1^{*} 5^{\wedge} 3^{*} 7^{\wedge} 0=1500$ & MAIOR \\
\hline $24=3.4 .2$ & 2 & 3 & 1 & 0 & $2^{\wedge} 2^{*} 3^{\wedge} 3^{*} 5^{\wedge} 1^{*} 7^{\wedge} 0=540$ & MAIOR \\
\hline $24=4.2 .3$ & 3 & 1 & 2 & 0 & $2^{\wedge} 3^{*} 3^{\wedge} 1^{*} 5^{\wedge} 2^{*} 7^{\wedge} 0=600$ & MAIOR \\
\hline $24=4.3 .2$ & 3 & 2 & 1 & 0 & $2^{\wedge} 3^{*} 3^{\wedge} 2^{*} 5^{\wedge} 1^{*} 7^{\wedge} 0=360$ & MENOR \\
\hline $24=3.8$ & 2 & 7 & 0 & 0 & $2^{\wedge} 2^{*} 3^{\wedge} 7^{*} 5^{\wedge} 0^{*} 7^{\wedge} 0=\ldots$ & MAIOR \\
\hline $24=4.6$ & 3 & 5 & 0 & 0 & $2^{\wedge} 3^{*} 3^{\wedge} 5^{*} 5^{\wedge} 0^{*} 7^{\wedge} 0=\ldots$ & MAIOR \\
\hline
\end{tabular}

Figura 3: Determinação do menor número que tem 24 divisores.

Analisando os resultados obtidos na tabela acima, conclui-se que o menor número que tem 24 divisores é o número 360, o que é coerente com a proposta de ter de escolher os maiores valores dos expoentes para os respectivos menores valores dos respectivos fatores primos, entre os números primos $2,3,5$ ou 7 .

Exemplo 3. Como proceder para determinar quantos números menores que 100 têm 12 divisores?

Neste caso, a decisão deve recair sobre todas as possíveis escolhas dos expoentes dos respectivos fatores primos 2,3 e 5 que satisfazem aos possíveis produtos $(m+1) \cdot(n+1) \cdot(p+1)=12$ que determinam números com 12 divisores que sejam menores que 100.

Aqui, também pode ser feita uma Mudança de Quadros para o quadro da Análise Combinatória e realizar uma avaliação acerca das possibilidades presentes nas "folhas terminais" de uma árvore de possibilidades, como mostrado na tabela a seguir, considerando que os valores indicados por MENOR (*) são os números menores que 100 que têm 12 divisores e, portanto, atendem à solução. 


\begin{tabular}{|c|c|c|c|c|c|}
\hline $\begin{array}{l}12 \text { escrito } \\
\text { em } 2 \text { ou } 3 \\
\text { produtos. }\end{array}$ & $\begin{array}{l}\text { Expoente } \\
\text { fator } \\
\text { primo } 2 \text {. }\end{array}$ & $\begin{array}{l}\text { Expoente } \\
\text { fator } \\
\text { primo } 3 .\end{array}$ & $\begin{array}{l}\text { Expoente } \\
\text { fator } \\
\text { primo } 5 .\end{array}$ & $\begin{array}{l}\text { Número obtido, } \\
\text { com } 12 \text { divisores. }\end{array}$ & Situação \\
\hline $12=2.2 .3$ & 1 & 1 & 2 & $2^{\wedge} 1^{*} 3^{\wedge} 1^{*} 5^{\wedge} 2=150$ & MAIOR \\
\hline $12=2.3 .2$ & 1 & 2 & 1 & $2^{\wedge} 1^{*} 3^{\wedge} 2^{*} * 5^{\wedge} 1=90$ & MENOR que 100(*) \\
\hline $12=3.2 .2$ & 2 & 1 & 1 & $2^{\wedge} 2^{*} 3^{\wedge} 1 * 5^{\wedge} 1=60$ & MENOR que 100(*) \\
\hline $12=1.12$ & 0 & 11 & 0 & $2^{\wedge} 0^{*} 3^{\wedge} 11^{*} 5^{\wedge} 0=\ldots \ldots$ & MAIOR \\
\hline $12=12.1$ & 11 & 0 & 0 & $2^{\wedge} 11^{*} 3^{\wedge} 0^{*} 5^{\wedge} 0=\ldots \ldots$ & MAIOR \\
\hline $12=2.6$ & 1 & 5 & 0 & $2^{\wedge} 1^{*} 3^{\wedge} 5^{*} 5^{\wedge} 0=486$ & MAIOR \\
\hline $12=6.2$ & 5 & 1 & 0 & $2^{\wedge} 5^{*} 3^{\wedge} 1^{*} 5^{\wedge} 0=96$ & MENOR que 100(*) \\
\hline $12=3.4$ & 2 & 3 & 0 & $2^{\wedge} 2^{*} 3^{\wedge} 3^{*} * 5^{\wedge} 0=108$ & MAIOR \\
\hline $12=4.3$ & 3 & 2 & 0 & $2^{\wedge} 3^{*} 3^{\wedge} 2^{*} * 5^{\wedge} 0=72$ & MENOR que 100(*) \\
\hline
\end{tabular}

Figura 4: Determinação dos números menores que 100 que têm 12 divisores.

Analisando os resultados da tabela acima, apenas os números 60, 72, 90 e 96 são os números menores que 100 que possuem 12 divisores.

Exemplo 4. O propósito é o de determinar o número de modos que se tem para iluminar uma sala que possui 4 lâmpadas, todas elas em perfeito estado de funcionamento, e fixas ao teto da sala. Considere as lâmpadas $L_{1}, L_{2}, L_{3}$ e $L_{4}$. De uma maneira simplificada, podemos obter a solução após utilizar a enumeração de todas as possibilidades de escolhas de uma ou mais lâmpadas, independentemente da posição da lâmpadas ou lâmpadas que estará (ao) acesa (s) e, em seguida, efetuar a contagem direta. A seguir, todas as possibilidades de iluminar a sala:

- iluminando a sala com uma única lâmpada: $L_{1}$ ou $L_{2}$ ou $L_{3}$ ou $L_{4}$, ou seja, 4 (quatro) possibilidades;

- iluminando a sala com duas lâmpadas: $L_{1}$ e $L_{2}$ ou $L_{1}$ e $L_{3}$ ou $L_{1}$ e $L_{4}$ ou $L_{2}$ e $L_{3}$ ou $L_{2}$ e $L_{4}$ ou $L_{3}$ e $L_{4}$, ou seja, 6 (seis) possibilidades;

- iluminando a sala com três lâmpadas: $L_{1}$ e $L_{2}$ e $L_{3}$ ou $L_{1}$ e $L_{2}$ e $L_{4}$ ou $L_{1}$ e $L_{3}$ e $L_{4}$ ou $L_{2}$ e $L_{3}$ e $L_{4}$, ou seja, 4 (quatro) possibilidades;

- iluminando a sala com todas as 4 (quatro) lâmpadas: $L_{1}$ e $L_{2}$ e $L_{3}$ e $L_{4}$, ou seja, 1 (uma) única possibilidade.

É possível obter a solução construindo uma árvore de possibilidades, tomando por base as ideias do Princípio Multiplicativo, da Análise Combinatória e, assim, por meio da enumeração de todas as possibilidades obteremse a solução. A figura a seguir apresenta a construção de 4 (quatro) árvores de possibilidades que, juntas, mostram todas as diferentes maneiras que se têm de iluminar uma sala que possui 4 (quatro) lâmpadas: 


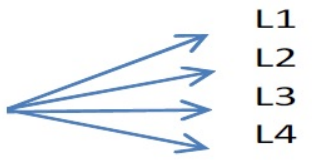

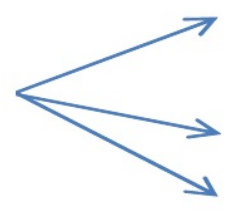

L1
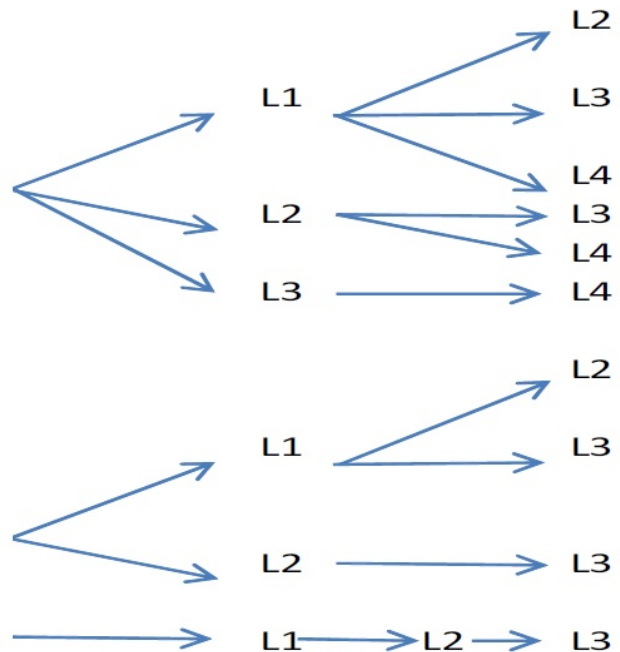

L1

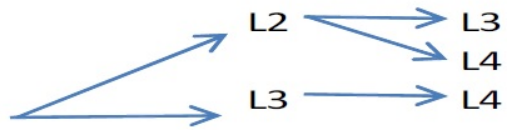

Total de possibilidades: 15
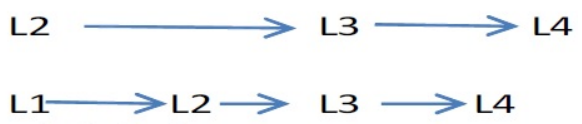

Figura 5: Árvore de possibilidades para obter a solução do exemplo 4.

Assim, por meio da enumeração de todas as possibilidades que compõem a solução do problema proposto, é feita a contagem direta e se obtêm as 15 (quinze) diferentes maneiras para iluminar uma sala que possui 4 (quatro) lâmpadas.

Uma Mudança de Quadros poderia ser feita de modo a provar, por outros argumentos, agora utilizando conceitos da Teoria dos Conjuntos, que o resultado do exemplo 4 é verdadeiro. Assim, Dado o conjunto $A=\left\{L_{1}, L_{2}, L_{3}, L_{4}\right\}$, o conjunto de partes desse conjunto é dado por:

$$
\begin{aligned}
\wp(A)= & \left\{\emptyset,\left\{L_{1}\right\},\left\{L_{2}\right\},\left\{L_{3}\right\},\left\{L_{4}\right\},\left\{L_{1}, L_{2}\right\},\left\{L_{1}, L_{3}\right\},\left\{L_{1}, L_{4}\right\},\left\{L_{2}, L_{3}\right\},\left\{L_{2}, L_{4}\right\},\left\{L_{3}, L_{4}\right\},\right. \\
& \left.\left\{L_{1}, L_{2}, L_{3}\right\},\left\{L_{1}, L_{2}, L_{4}\right\},\left\{L_{1}, L_{3}, L_{4}\right\},\left\{L_{2}, L_{3}, L_{4}\right\},\left\{L_{1}, L_{2}, L_{3}, L_{4}\right\}\right\}
\end{aligned}
$$

A quantidade de elementos do conjunto de partes é dada por $\# \wp(A)=2^{4}=16$. Como deve haver ao menos 1 (uma) lâmpada acesa de modo que a sala fique iluminada, o conjunto $\emptyset$ não deve ser considerado em função de representar a ideia de que todas as lâmpadas estão desligadas. Assim, tem-se um total de $2^{4}-1=15$ possibilidades para iluminar a sala. Essa resolução permitir fazer uma ampliação conceitual e determinar o total de maneira de iluminar uma sala com $k$ lâmpadas.

É possível fazer uma nova Mudança de Quadros para provar, de outro modo, que o resultado acima é verdadeiro, agora utilizando propriedades do Triângulo de Pascal. Sabe-se que o somatório dos elementos da $n$-ésima linha do Triângulo de Pascal é dado por $2^{n}$. No caso particular deste exemplo, considerando a $4^{\underline{a}}$ linha do triângulo de Pascal, uma vez que se dispõe de 4 lâmpadas na sala, esse somatório é dado por $2^{4}=16=c_{4,0}+c_{4,1}+c_{4,2}+c_{4,3}+c_{4,4}$. Cada uma das parcelas pode ser entendida como $c_{4, p}$, ou seja, o número de modos de acender $p$ lâmpadas entre 4 
lâmpadas disponíveis, onde $p \in\{0,1,2,3,4\}$. O coeficiente binomial $c_{4,0}$ indica a opção de, entre as 4 lâmpadas disponíveis, não ter escolhido acender nenhuma delas, o que, evidentemente, não atende à solução do problema. Assim, esta opção não deve ser considerada. Portanto, o total de possibilidades é dado por $16-1=15$.

Ainda dentro do quadro da análise combinatória, prova-se que o resultado acima é verdadeiro, novamente utilizando conceitos próprios da Análise Combinatória: o Princípio Multiplicativo.

Há 2 (duas) possibilidades para a lâmpada $L_{1}$ : estar acesa ou apagada. $\mathrm{O}$ mesmo se aplica para as demais lâmpadas da sala. Assim, o total de possibilidades é dado por $2 \cdot 2 \cdot 2 \cdot 2=16$ possibilidades. Mas, dentre essas 16 possibilidades, incluímos a possibilidade de que as 4 (quatro) lâmpadas estivessem todas apagadas. Logo, desse total de 16 possibilidades, é preciso retirar essa possibilidade (todas as lâmpadas apagadas) ficando, então, com $16-1=15$ distintos modos de ter a sala acesa, quando se dispõe de 4 lâmpadas.

Esse resultado pode ser generalizado para um quantitativo de n lâmpadas disponíveis em uma sala, quando se quer calcular o total de diferentes modos de iluminar essa sala, o que dá um total de $\left(2^{n}-1\right)$ diferentes modos de a sala ficar iluminada.

Segundo Teixeira (2012), situações-problema de contagem com as que foram apresentadas acima não são comuns de ser desenvolvidas por professores da Educação Básica em sala de aula. Assim se refere Teixeira (2012) acerca dessa questão: ,

Ademais, são raros os livros didáticos que sinalizam para a motivação quanto à mobilização de diferentes estratégias para a resolução de um mesmo problema de contagem uma vez que, grande parte deles apresenta a resolução de situações-problema exemplos por meio da aplicação direta de uma fórmula que, em geral, foi somente apresentada de início. Além disso, também não identificamos nos livros didáticos a preocupação de orientar o aluno de que não há necessidade do uso de uma fórmula para determinar a solução para um problema de contagem por meio da apresentação de soluções para problemas de contagem que explorem o uso do Princípio Multiplicativo, do Princípio Aditivo e por meio de uma representação, como a árvore de possibilidades, sem o uso de uma fórmula em prosseguimento (Teixeira, 2012, p. 391-392)

Exemplo 5. Avaliamos, a seguir, uma Mudança de Quadro presente em conceitos próprios da Álgebra Linear. Considere o sistema linear

$$
\begin{aligned}
& a_{11} x_{1}+\cdots+a_{1 n} x_{n}=b_{1} \\
& a_{m 1} x_{1}+\cdots+a_{m n} x_{n}=b_{m}
\end{aligned}
$$

A matriz ampliada desse sistema (1) é formada pela matriz dos coeficientes das variáveis e a matriz dos termos independentes:

$$
\left[\begin{array}{cccc}
a_{11} & \cdots & a_{1 n} & b_{1} \\
\vdots & \ddots & \vdots & \vdots \\
a_{m 1} & \cdots & a_{m n} & b_{m}
\end{array}\right]
$$

Assim, o sistema linear pode ser representado pelo produto matricial $A \cdot X=B$, onde $A_{m \times n}$ é a 
$\operatorname{matriz}\left[\begin{array}{ccc}a_{11} & \cdots & a_{1 n} \\ \vdots & \ddots & \vdots \\ a_{m 1} & \cdots & a_{m n}\end{array}\right], B_{m \times 1}$ é a matriz $\left[\begin{array}{c}b_{1} \\ \vdots \\ b_{m}\end{array}\right]$ e $X_{n \times 1}$ é a matriz $\left[\begin{array}{c}x_{1} \\ \vdots \\ x_{n}\end{array}\right]$. O sistema linear acima também pode ser representado por meio da equação vetorial

$$
X_{1}\left(a_{11}, a_{21}, \ldots, a_{m 1}\right)+\cdots+X_{n}\left(a_{1 n}, a_{2 n}, \ldots, a_{m n}\right)=\left(b_{1}, \ldots, b_{m}\right) .
$$

A matriz $A_{m \times n}$ é vista como uma aplicação linear $T_{A}: \mathbb{R}^{n} \rightarrow \mathbb{R}^{m}$ em relação às bases canônicas $\beta=\left\{v_{1}, \ldots, v_{n}\right\}$ de $\mathbb{R}^{n}$ e $\beta^{\prime}=\left\{w_{1}, \ldots, w_{m}\right\}$ de $\mathbb{R}^{m}$. Diz-se, também, que podemos associar a matriz $A_{m \times n}$ como:

$$
\begin{aligned}
T_{A}: \mathbb{R}^{n} & \rightarrow \mathbb{R}^{m} \\
v & \mapsto T_{A}(v) .
\end{aligned}
$$

Seja $X=[v]_{\beta}=\left[\begin{array}{c}x_{1} \\ \vdots \\ x_{n}\end{array}\right]$ e $T_{A}(v)=A \cdot X=\left[\begin{array}{ccc}a_{11} & \cdots & a_{1 n} \\ \vdots & \ddots & \vdots \\ a_{m 1} & \cdots & a_{m n}\end{array}\right] \cdot\left[\begin{array}{c}x_{1} \\ \vdots \\ x_{n}\end{array}\right]=\left[\begin{array}{c}y_{1} \\ \vdots \\ y_{n}\end{array}\right]$. Então, $T_{A}(v)=$ $y_{1} w_{1}+\cdots+y_{m} w_{m}$, onde $y_{i}=A_{i} \cdot X$ e $A_{i}$ é a $i$-ésima linha de $A$. Em geral,

$$
\begin{aligned}
& T_{A}(u+v)=A \cdot(u+v)=A u+A v=T_{A}(u)+T_{A}(v) \\
& T_{A}(\alpha v)=A \cdot(\alpha v)=\alpha(A u)=\alpha T_{A}(u) .
\end{aligned}
$$

Logo,

$$
\begin{aligned}
T_{A}: \mathbb{R}^{n} & \rightarrow \mathbb{R}^{m} \\
v & \mapsto T_{A}(v) .
\end{aligned}
$$

é uma transformação linear. Assim, dada uma matriz $A_{m \times n}$, ela produz uma transformação linear

$$
\begin{aligned}
T_{A}: \mathbb{R}^{n} & \rightarrow \mathbb{R}^{m} \\
v & \mapsto T_{A}(v) .
\end{aligned}
$$

em relação às bases canônicas $\beta=\left\{v_{1}, \ldots, v_{n}\right\}$ de $\mathbb{R}^{n}$ e $\beta^{\prime}=\left\{w_{1}, \ldots, w_{m}\right\}$ de $\mathbb{R}^{m}$.

Neste caso, fez-se uma Mudança de Quadro associado aos conceitos presentes na Teoria das Matrizes e dos Sistemas Lineares para o quadro da Álgebra Linear, particularmente, para o estudo das Transformações Lineares.

Por outro lado, dada uma transformação linear $T: \mathbb{R}^{n} \rightarrow \mathbb{R}^{m}$, ela pode ser representada por uma matriz $[T]_{\beta^{\prime}}^{\beta}$, relativamente às bases $\beta=\left\{v_{1}, \ldots, v_{n}\right\}$ e $\beta^{\prime}=\left\{w_{1}, \ldots, w_{m}\right\}$, respectivamente, de $\mathbb{R}^{n}$ e de $\mathbb{R}^{m}$, tal que

$$
\begin{gathered}
T\left(v_{1}\right)=u_{1}=\sum_{i=1}^{m} a_{i 1} \cdot w_{i} \\
\vdots \\
T\left(v_{n}\right)=u_{n}=\sum_{i=1}^{m} a_{i m} \cdot w_{i}
\end{gathered}
$$


Daí: $[T]_{\beta^{\prime}}^{\beta}=\left[\begin{array}{ccc}a_{11} & \cdots & a_{1 m} \\ \vdots & \ddots & \vdots \\ a_{n 1} & \cdots & a_{n m}\end{array}\right]$ é a matriz de $T$ em relação às bases canônicas $\beta=\left\{v_{1}, \ldots, v_{n}\right\}$ de $\mathbb{R}^{n}$ e $\beta^{\prime}=\left\{w_{1}, \ldots, w_{m}\right\}$ de $\mathbb{R}^{m}$. Ademais, a matriz $c$ é a transposta da matriz dos coeficientes do sistema linear apresentado acima, ou seja, $A_{m \times n}=\left[\begin{array}{ccc}a_{11} & \cdots & a_{1 n} \\ \vdots & \ddots & \vdots \\ a_{m 1} & \cdots & a_{m n}\end{array}\right]$. Portanto, $T$ passa a ser a aplicação linear associada à matriz $A$ e bases $\beta=\left\{v_{1}, \ldots, v_{n}\right\}$ e $\beta^{\prime}=\left\{w_{1}, \ldots, w_{m}\right\}$, isto é, $T=T_{A}$. Portanto, as propriedades que a matriz $A_{m \times n}$ possui estão diretamente associadas às propriedades da transformação linear associada a ela, como por exemplo:

- $\operatorname{dim}(\operatorname{Im}(T))=p=$ posto da matriz $[T]_{\beta^{\prime}}^{\beta}=$ número de linhas não nulas da matriz $B_{m \times n}$, a matriz-linha reduzida à forma escada linha equivalente a $A_{m \times n}$.

- $\operatorname{dim}(\operatorname{Ker}(T))=\operatorname{dim}(N u c(T))=$ nulidade da matriz $[T]_{\beta^{\prime}}^{\beta}=n-p=$ diferença entre o número de colunas da matriz $A_{m \times n}$ e o posto da matriz $[T]_{\beta^{\prime}}^{\beta}$.

- a transformação linear $T: V \rightarrow W$ é inversível se e somente se $\operatorname{det}\left([T]_{\beta^{\prime}}^{\beta}\right) \neq 0$.

- se a transformação linear $T: V \rightarrow W$ é inversível então $T^{-1}: W \rightarrow V$ é operador linear e $\left[T^{-1}\right]_{\beta}^{\beta^{\prime}}=$ $\left([T]_{\beta^{\prime}}^{\beta}\right)^{-1}$.

Essa Mudança de Quadro, presente na Teoria das Matrizes e na Álgebra Linear, permite compreender que todo o estudo dos operadores lineares, aí associados os conceitos relativos ao estudo dos autovalores e dos autovetores; os operadores auto-adjuntos e ortogonais; as formas lineares, bilineares e quadráticas, e a classificação de cônicas e quádricas, podem ser feitas com a utilização das ferramentas matemáticas associadas à Teoria das Matrizes e à Teoria dos Determinantes, configurando-se em valioso instrumento para a exploração de conceitos que estão presentes nas cônicas, seções cônicas e nas quádricas, por exemplo, por meio de conceitos algébricos, em estreita correlação com os fundamentos da geometria que foram estudadas pelos gregos, e desenvolvidas a partir de suas propriedades geométricas, presentes nos trabalhos de Apollonius (260-170 A.C.). As considerações teóricas acerca dos conceitos próprios da álgebra Linear estão em [2].

Exemplo 6. Outra situação de Mudança de Quadros se configura no caso de determinar o total de diagonais de um polígono regular de $n$ lados, $n \geq 3$.

Escolhido um vértice qualquer $v_{1}$, os segmentos de reta que unem os vértices adjacentes a esse vértice $\left(v_{2}\right.$ e $\left.v_{n}\right)$ são lados do polígono e, assim, não podem ser contados como diagonais. Sobram $(n-3)$ vértices do polígono cujos segmentos de reta que os unem ao vértice $v_{1}$ são diagonais do polígono. Portanto, a partir do vértice $v_{1}$ se pode traçar $(n-3)$ diagonais. $\mathrm{O}$ mesmo ocorre para quaisquer um dos outros $(n-1)$ vértices do polígono. Assim, é possível traçar um subtotal de $(n-3) \cdot n$ diagonais. Mas, todas as diagonais assim consideradas foram duplamente contadas quando se conta as diagonais a partir de cada um dos vértices nas extremidades dessas diagonais, o que evidencia uma contagem em dobro. De modo a obter a contagem real, divide-se esse total por 2. Assim, há $d=\frac{(n-3) \cdot n}{2}$ diagonais em um polígono regular de $n$ vértices (correspondentemente, $n$ lados), $n \geq 3$.

Vamos fazer uma Mudança de Quadros para provar, de outro modo, que o resultado acima é verdadeiro no quadro da análise combinatória: Se um polígono regular tem n lados, ao escolher 2 
(dois) quaisquer de seus vértices e uni-los por meio de um segmento de reta, é possível traçar uma diagonal ou um lado do polígono. Por outro lado, o número total de modos de fazer a escolha de 2 (dois) quaisquer vértices é calculado por $C_{n, 2}$ (número de modos de escolher 2 (dois) objetos dentre $n$ objetos distintos dados (combinações simples dois a dois)). Assim, o total $C_{n, 2}$ de escolhas define, unicamente, o total de diagonais acrescido do número de lados do polígono de $n$ vértices, ou seja: $C_{n, 2}=$ número de diagonais $(d)+$ número de lados $(n)$. Portanto, $d=C_{n, 2}-n=\frac{n \cdot(n-1)}{2}-n=$ $\frac{n \cdot(n-3)}{2}, n \geq 3$.

Uma nova Mudança de Quadros poderia ser feita para provar, por Indução no número de vértices (respectivamente lados), que o resultado acima é verdadeiro.

O polígono regular com o menor número de vértices é o triângulo, que possui 3 vértices. Portanto, o triângulo não possui diagonais. Assim, vale a primeira propriedade de indução $P_{1}$, pois, para $n=3$ tem-se que $d_{3}=\frac{3 \cdot(3-3)}{2}=0$.

Considere, por hipótese de indução, que o resultado acima é válido para $n=k$. Assim, $d_{k}=\frac{k \cdot(k-3)}{2}$.

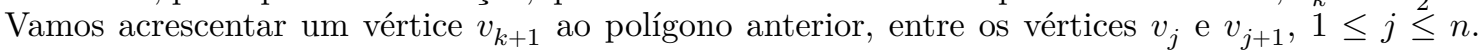
Desse novo vértice traçam-se $k-2$ novas diagonais (exceto para os vértices vizinhos ao vértice $v_{k+1}$, que são os vértices $v_{j}$ e $v_{j+1}$ ), e mais uma diagonal (a diagonal que conecta os vértices $v_{j} \mathrm{e}$ $v_{j+1}$. No polígono anterior, ao conectar os vértices $v_{j}$ e $v_{j+1}$ forma-se um lado do novo polígono. Assim, no novo polígono, tem-se $(k-2)+1=k-1$ diagonais a mais que o total de diagonais presentes no polígono anterior. Logo:

$$
d_{k+1}=(k-1)+\frac{k \cdot(k-3)}{2}=\frac{2 k-2+k^{2}-3 k}{2}=\frac{k^{2}-k-2}{2}=\frac{(k-2) \cdot(k-1)}{2}=\frac{(k+1) \cdot[(k+1)-3]}{2} .
$$

Portanto, o resultado fica provado, por indução.

Outra situação de Mudança de Quadros, agora no quadro da análise combinatória, de modo que se queira estender a ideia anterior para um poliedro regular de n vértices, de maneira a determinar o total de diagonais internas de poliedros regulares.

Ao escolher 2 (dois) quaisquer de seus vértices (cuja totalidade é dada por $C_{n, 2}$ ) e uni-los por meio de um segmento de reta, pode acarretar uma das três situações seguintes: ou se obtém uma diagonal de face, ou uma diagonal interna do poliedro ou uma aresta do poliedro. Assim, $C_{n, 2}=$ número de diagonais de face $(D F)+$ número de diagonais internas $(D I)+$ número de arestas $(A)$. Logo: número de diagonais internas $(D I)=C_{n, 2}-$ número de diagonais de face $(D F)$ - número de arestas $(A)$, ou seja: $(D I)=C_{n, 2}-(D F)-(A)$. Vejamos o seguinte exemplo: Quantas diagonais internas possui um octaedro?

As 8 (oito) faces do octaedro são formadas por triângulos equiláteros que, obviamente, não têm diagonais de face. Além disso, o octaedro possui 6 vértices e 12 arestas (as quatro arestas da parte de cima, as quatro da parte de baixo e as quatro ao redor dos quatro vértices na parte central). Assim: $(D I)=C_{6,2}-0-12=15-0-12=3$, ou seja, o octaedro possui 3 (três) diagonais internas, que são as seguintes: a diagonal que une os dois vértices isolados e as duas diagonais que unem, dois a dois, os vértices que estão na parte central.

De modo similar, prova-se que um tetraedro não possui diagonais internas, pois: $(D I)=C_{4,2}-0$ (as faces são triangulares) - 6 (as três arestas que ligam os vértices da base ao vértice de cima mais as três arestas que ligam os três vértices da base) $=6-0-6=0$. O quadro abaixo mostra o total de diagonais internas para cada um dos Poliedros de Platão: 


\begin{tabular}{|c|c|c|c|c|c|c|c|c|}
\hline Poliedro & $\begin{array}{l}\text { número de } \\
\text { vértices }(\mathrm{V})\end{array}$ & $\begin{array}{l}\text { número } \\
\text { de faces }\end{array}$ & $\begin{array}{l}\text { polígono } \\
\text { das faces }\end{array}$ & $\begin{array}{c}\text { número de diago- } \\
\text { nais por face }\end{array}$ & $\begin{array}{l}\text { total de diagonais } \\
\text { de face (DF) }\end{array}$ & $\begin{array}{c}\text { número } \\
\text { de arestas }(A)\end{array}$ & $\mathrm{C}_{\mathrm{V}, 2}$ & $\begin{array}{c}\mathrm{D}=\mathrm{C} \mathrm{V}, 2-\mathrm{A}-\mathrm{DF} \\
\text { diagonais internas }\end{array}$ \\
\hline Tetraedro & 4 & 4 & triângulo & 0 & $4 \times 0=0$ & $(4 \times 3) / 2=6$ & 6 & 0 \\
\hline Octaedro & 6 & 8 & triângulo & 0 & $8 \times 0=0$ & $(8 \times 3) / 2=12$ & 15 & 3 \\
\hline Cubo & 8 & 6 & quadrado & 2 & $6 \times 2=12$ & $(6 \times 4) / 2=12$ & 28 & 4 \\
\hline \begin{tabular}{|l|} 
Dodecaedro \\
\end{tabular} & 20 & 12 & pentágond & 5 & $12 \times 5=60$ & $(12 \times 5) / 2=30$ & 190 & 100 \\
\hline Icosaedro & 12 & 20 & triângulo & 0 & $20 \times 0=0$ & $(20 \times 3) / 2=30$ & 435 & 405 \\
\hline
\end{tabular}

Figura 6: Determinação do total de diagonais internas dos Poliedros de Platão.

Como ampliação das ideias conceituais tratadas se pode determinar uma maneira de contabilizar o número total de diagonais internas de um prisma regular reto que possui em suas bases um polígono regular de $n$ lados.

Podemos fazer assim: Esse prisma possui $n$ retângulos, congruentes entre si, que formarão as $n$ faces laterais do prisma. Como cada retângulo possui 2 (duas) diagonais tem-se, então, um subtotal de $2 n$ diagonais de face, contabilizadas no total de faces laterais do prisma. As 2 (duas) faces (superior e inferior) são formadas por um polígono regular de $n$ lados, $n \geq 3$ e portanto, cada uma das faces possui um total de $d=\frac{(n-3) \cdot n}{2}$ diagonais.

Assim, há um subtotal de $(n-3) \cdot n$ diagonais de face contabilizadas nas 2 (duas) bases. Portanto, tem-se o total de $2 n+(n-3) \cdot n$ diagonais de face em um prisma regular reto que tem em suas bases um polígono regular de $n$ lados.

Qual o total de diagonais internas desse prisma regular reto?

Inicialmente vamos numerar os $n$ vértices da face superior e os $n$ vértices da face inferior obedecendo à mesma ordem dentre os índices desses vértices, isto é, para um dado vértice $v_{j}$ da face superior, a aresta que o conecta com a face inferior encontra, exatamente, logo abaixo, o vértice $w_{j}$. Assim, escolhido um vértice qualquer $v_{j}$ da face superior, os segmentos de reta que unem os vértices da face inferior "próximos deste vértice" - que são os vértices adjacentes $w_{j-1}, w_{j}$ e $w_{j+1}$ - quando conectados ao vértice $v_{j}$ definem as seguintes situações: uma aresta $\left(v_{j}, w_{j}\right)$ ou então as diagonais de face $\left(v_{j}, w_{j-1}\right)$ e $\left(v_{j}, w_{j+1}\right)$ (lembre que essas diagonais já foram incluídas na contagem anterior). Portanto, escolhido um vértice $v_{j}$ na face superior, há $(n-3)$ vértices na face inferior que, quando conectados ao vértice $v_{j}$ da face superior, definem diagonais internas. Portanto, contabilizando o total de diagonais internas para todos os vértices da face superior, há um total de $n \cdot(n-3)$ diagonais internas.

Se repetíssemos o procedimento para os vértices localizados na face inferior, estaríamos contando novamente essas mesmas diagonais internas. Então, há um total de $2 n+(n-3) \cdot n$ diagonais de face e $n \cdot(n-3)$ diagonais internas, totalizando $2[n+n \cdot(n-3)]=2\left[n^{2}-2 n\right]=2 n \cdot[n-2]$ diagonais de um prisma, cujas bases são formadas por polígonos regulares de $n$ vértices.

Ainda dentro do quadro da análise combinatória, é possível provar que o resultado acima é verdadeiro.

Se o prisma regular tem $2 n$ vértices, ao escolher 2 (dois) quaisquer de seus vértices e uni-los por meio de um segmento de reta, podemos obter uma das três situações: uma diagonal de face, uma aresta ou uma diagonal interna desse poliedro. Por outro lado, o número de modos de fazer a escolha de 2(dois) quaisquer vértices, como visto, é determinado por $C_{2 n, 2}$ (número de modos de 
escolher 2 (dois) objetos dentre os $2 n$ objetos distintos dados (combinações simples)). Assim, esse total de escolhas $\left(C_{2 n, 2}\right)$ define, unicamente, o total de diagonais de face, acrescido do total de arestas e acrescido do total de diagonais internas desse poliedro. Ou seja: $C_{2 n, 2}=$ número de diagonais de face $(D F)+$ número de arestas $(A)+$ número de diagonais internas $(D I)$. Portanto,

$$
D I+D F=C_{2 n, 2}-A .
$$

O número total de arestas é calculado assim: $n$ arestas que conectam os vértices da face inferior aos vértices da face superior, mais as $2 n$ arestas (que são os lados dos 2 (dois) polígonos regulares das faces superior e inferior) dos 2 (dois) polígonos regulares que constituem o prisma, totalizando $3 n$ arestas. Assim, de (4) tem-se:

$$
D I+D F=\frac{2 n(2 n-1)}{2}-3 n=\frac{4 n^{2}-2 n-6 n}{2}=\frac{4 n^{2}-8 n}{2}=\frac{2 n^{2}-4 n}{1}=2 n(n-2) .
$$

Se quiséssemos calcular o total de diagonais internas $(D I)$, poderíamos, utilizando a igualdade (5), proceder assim: Primeiramente vamos determinar o total de diagonais de face: As faces são de dois tipos: retângulos (as faces laterais) e polígonos regulares de $n$ lados (a face superior e a face inferior do prisma). Nos retângulos, há um total de: $n \cdot\left[C_{4,2}-4\right]=n \cdot[6-4]=2 n$ diagonais de face. Nos polígonos regulares (faces superior e inferior) há um total de: $2 \cdot \frac{(n-3) \cdot n}{2}=n \cdot(n-3)=n^{2}-3 n$ diagonais de face. Logo, o total de diagonais de face é dado por

$$
D F=2 n+n^{2}-3 n=n^{2}-n .
$$

Portanto, de (5) e (6), tem-se que:

$$
D I=2 n \cdot(n-2)-\left(n^{2}-n\right)=2 n^{2}-4 n-n^{2}+n=n^{2}-3 n=n \cdot(n-3),
$$

como provado antes.

\section{Considerações finais}

Com frequência uma Mudança de Quadros vem acompanhada de uma mudança de registro. Uma mudança de registro coincide, em muitos casos, com uma Mudança de Quadro. Nas situaçõesproblema que foram apresentadas identificam-se diferentes registros de representação semiótica ${ }^{1}$, alguns deles dentro do mesmo quadro - da Análise Combinatória - e outros nos quadros da Teoria dos Conjuntos, da Teoria das Matrizes e da Álgebra Linear. A mudança de resolução, em qualquer uma das situações apresentadas, para o caso inicial ou vice-versa, representa uma Mudança de Quadros. Quando se obtém, em uma mesma situação-problema, uma Mudança de Quadros, e, a seguir, por uma nova Mudança de Quadros se retorna ao quadro anterior, dizse que fizemos uso de um Jogo de Quadros.

Para descrever uma Mudança de Quadro é preciso se centrar essencialmente sobre uma situaçãoproblema enquanto que, para descrever uma mudança de registro é preciso se centrar essencialmente sobre os objetos que estão sendo representados.

\footnotetext{
${ }^{1}$ Raymond Duval, filósofo e psicólogo francês, fornecem-nos um referencial estruturado de análise do funcionamento cognitivo da compreensão em matemática através da Teoria dos Registros de Representações Semióticas. Os objetos matemáticos não são diretamente acessíveis à percepção, o seu acesso passa, necessariamente, por representações semióticas (formas sob a qual a informação é descrita).
} 
Assim, segundo Raymond Duval ([7]), a compreensão conceitual sobre o objeto matemático função, por exemplo, só é alcançada quando se coordenam os registros algébricos, tabular e gráfico. E esta é a condição para que o objeto não seja confundido com a sua representação.

Por essa razão considera-se que a passagem entre registros não se constitui em opção pedagógica do professor que permita enriquecer a resolução de uma situação-problema, mas é desejável que ela deva ser considerada como uma tarefa imprescindível e obrigatória para encaminhar o processo de ensino aprendizagem.

$\mathrm{O}$ autor deste artigo entende que a passagem de registros se trata de um momento da aprendizagem que não pode ser deixado de lado pelos professores, mas que cada um deve considerá-lo como uma tarefa docente necessária e obrigatória para se atingir uma aprendizagem significativa.

Por essas razões entende que para encaminhar uma aprendizagem efetiva, não há um acesso direto ao objeto matemático quando este é apresentado separado das suas representações quando se procura oferecer aos alunos um conhecimento abrangente e aprofundado do objeto matemático em questão.

Com as considerações que foram apresentadas neste trabalho - estendendo-se também para outros conceitos matemáticos -, o autor considera que Mudanças de Quadros devem ser incorporadas ao trabalho docente do professor. E este, por sua vez, também precisa incentivar seus alunos a fazerem o mesmo quando propuser que eles resolvam situações-problema pertinentes ao desenvolvimento de conceitos matemáticos em quadros diferentes da Matemática.

Práticas como a que aqui foi apresentada têm o propósito de incentivar a ampliação conceitual de alunos e professores, construindo pontes entre diferentes conceitos da Matemática.

\section{Referências}

[1] Almouloud, S. A. Fundamentos da Didática da Matemática. Curitiba. Editora UFPR, 2007.

[2] Boldrini, J. L., Costa, S. I. R., Figueiredo, V. L., Wetzler, H. G. Álgebra Linear. Harper \& Row do Brasil, 1980.

[3] Douady, R. Jeux de Dadres et Dialectique Outil-Objet dans l'Enseignement des Mathématiques. Tese (Doutorado), 1986.

[4] Douady, R. Jeux de Cadres et Dialectique Outil-Objet. Recherches en Didactique dês Mathématiques (RDM), vol. 7.2, p. 5-31, 1986.

[5] Douady, R. Repères. IREM, n. 6, Janvier, 1992.

[6] Douady, R. L'ingènierie Didactique, Um Moyen pour l'Enseignant d'Organiser lês Rapports Entie l'Enseignement et l'Apprentissage. Chaier de DIDIREM, n. 19, IREM, Université Paris VII, 1993.

[7] Duval, R. Semiósis e O Pensamento Humano. São Paulo: Livraria da Física, 2009.

[8] Rogalski, M. Les Chagements de Cadre dans La Pratique dês Mathématiquas et Le Jeu de Cadre de Règine Douady. In: Actes de La jounée em Hommage à Règine Douady, Université Paris 7, 2001.

[9] Teixeira, P. J. M. Um Estudo sobre Os Conhecimentos Necessários ao Professor de Matemática para A Exploração de Problemas de Contagem no Ensino Fundamental. Tese (Doutorado em Educação Matemática), UNIBAN (Universidade Bandeirante de São Paulo), São Paulo, 2012. 
Paulo Teixeira

IME-UFF, Colégio Pedro II

<pjuff@yahoo.com.br>

Recebido: 2015

Publicado: 2015 